\title{
Indirect Doppler flow systolic blood pressure measurements taken with and without headphones in privately-owned, conscious dogs
}

\author{
India R Gill ${ }^{1}$, Joshua M Price ${ }^{2}$, Jacqueline C Whittemore ${ }^{\text {Corresp. } 1}$ \\ 1 Department of Small Animal Clinical Sciences, University of Tennessee - Knoxville, Knoxville, Tennessee, United States \\ 2 Office of Information Technology, University of Tennessee - Knoxville, Knoxville, Tennessee, United States \\ Corresponding Author: Jacqueline C Whittemore \\ Email address: jwhittemore@utk.edu
}

Objective. The purpose of this study was to assess the effect of headphone use and covariates on indirect radial Doppler flow systolic arterial blood pressure (BP) measurements in dogs.

Methods. Between May and August 2018, 100 privately-owned dogs were enrolled. Blood pressure was measured in lateral recumbency, with and without headphones, using a randomized crossover design. The initial BP, mean of BP 2-6, weight, BCS, MCS, anxiety score, and heart rate were recorded. Mixed effects crossover analyses and Spearman rank correlation coefficients were determined.

Results. Eighty-four dogs completed the study. Eleven dogs were removed due to excessive anxiety, 10 of which were in the non-headphone first group. The number of dogs diagnosed as hypertensive did not differ between measurement types (19 vs 18), with 7 dogs categorized as hypertensive during both periods. Significant differences in BP were identified $(F[1,80]=4.3, P=0.04)$ due to higher results for measurements taken without headphones for BP 1, but not BP 2-6. Systolic BP was positively correlated with anxiety score, age, and weight.

Conclusions and Clinical Relevance. Though BP 1 was significantly higher when taken without headphones, this pattern did not persist for BP 2-6. Lack of association between BP 2-6 results and measurement type could reflect exclusion of dogs most sensitive to white coat hypertension, acclimation to technique, or improved sound quality of headphones. Given significantly higher BP 1 results and disproportionate exclusion of dogs due to anxiety when measurements first were taken without headphones, use of headphones is recommended to improve accuracy of results. 
1 Indirect Doppler flow systolic blood pressure measurements taken with and without

2 headphones in privately-owned, conscious dogs

3 India R. Gill, BS; ${ }^{1}$ Joshua M. Price, MS; ${ }^{2}$ Jacqueline C. Whittemore, DVM, PhD, DACVIM.1

4

$5{ }^{1}$ Department of Small Animal Clinical Sciences, University of Tennessee - Knoxville, Knoxville,

6 Tennessee, United States

$7 \quad$ 2Office of Information Technology, University of Tennessee - Knoxville, Knoxville, Tennessee,

8 United States

9

10 Corresponding Author:

11 Jacqueline Whittemore ${ }^{1}$

12 Department of Small Animal Clinical Sciences, College of Veterinary Medicine, University of

13 Tennessee, 2407 River Drive, Knoxville, TN 37996, United States

14 Email address: jwhittemore@utk.edu 


\section{Abstract}

Objective. The purpose of this study was to assess the effects of headphone use and possible covariates on indirect radial Doppler flow systolic arterial blood pressure (BP) measurements in 21 dogs.

Methods. Between May and August 2018, 100 privately-owned dogs were enrolled. Blood pressure was measured in lateral recumbency, with and without headphones, using a randomized crossover design. The initial BP, mean of BP 2-6, weight, body and muscle condition scores, anxiety score, and heart rate were recorded. Mixed effects crossover analyses and Spearman rank correlation coefficients were determined.

Results. Eighty-three dogs completed the study. Thirteen dogs were excluded due to excessive anxiety, 12 of which were in the non-headphone first group. The number of hypertensive dogs did not differ between measurement types (19 vs 18); 7 dogs were categorized as hypertensive during both periods. Significant differences in BP were identified due to higher results for measurements taken without headphones for BP $1(\mathrm{~F}[1,80]=4.3, P=0.04)$, but not BP 2-6. Systolic BP was positively correlated with anxiety score, age, and weight.

Conclusions and Clinical Relevance. Though BP 1 was significantly higher when taken without headphones, this pattern did not persist for BP 2-6. Lack of association between BP 2-6 results and measurement type could reflect exclusion of dogs most sensitive to white coat hypertension, acclimation to technique, or other factors. Given significantly higher BP 1 results and disproportionate exclusion of dogs due to severe anxiety when measurements first were taken without headphones, use of headphones is recommended to improve accuracy of results. 


\section{Introduction}

Excitement or anxiety associated with the process of veterinary evaluation and blood pressure measurement can activate the central nervous system and increase systolic blood pressure, a phenomenon known as the white-coat effect or white-coat hypertension (Belew et al. 1999; Bragg et al. 2015; Höglund et al. 2012). Habituation has been associated with mixed effects on blood pressure measurements in cats. Although there was no significant difference in white-coat effect among 5 simulated office visits for healthy research cats undergoing repeated indirect oscillometric blood pressure measurement after physical examination, the magnitude of the white-coat effect was lowest during the first visit for $3 / 6$ cats (Belew et al. 1999). Failure to recognize the white-coat effect can result in unnecessary life-long treatment (Acierno et al. 2018) and potentially result in iatrogenic systemic hypotension. Conversely, failure to detect true hypertension can result in delayed diagnosis and, thus, progression of end-organ damage. As such, accurate blood pressure determination is essential to identify the presence of hypertension and need for intervention (Höglund et al. 2012).

The American College of Veterinary Internal Medicine has developed and recently updated guidelines for appropriate collection of indirect blood pressure measurements (Acierno et al. 2018). These include acclimating the patient to the room, equipment, and personnel; taking care in proper patient positioning and crystal placement; using an appropriately sized cuff and discarding outlier values; and having an experienced individual collect repeated measurements (Rondeau et al. 2013). Some professionals recommended that headphones be used for collection of measurements using the indirect Doppler flow technique lest noise from the machine startle the patient (Caney 2007; Whittemore et al. 2017). However, measurements are routinely taken without headphones as the importance of this recommendation has not been confirmed. One 
62 abstract reported no significant differences in indirect Doppler flow measurement results

63 collected with and without headphones 6 weeks apart in cats (Williams et al. 2010). Data

64 regarding the impact of headphone use on measurements taken in dogs are lacking.

65 The purpose of this study was to assess the impact of the use of headphones on indirect

66 systolic blood pressure measurements taken using the Doppler method in privately-owned dogs.

67 We hypothesized that indirect blood pressure measurements taken with and without the use of

68 headphones would be discordant, with higher results for measurements taken without

69 headphones.

70

71 Materials \& methods

72 Study Population - This study was conducted at the University of Tennessee's

73 Veterinary Medical Center and was approved by the Institutional Animal Care and Use

74 Committee of the University of Tennessee, Knoxville (protocol number 2428).

Privately-owned dogs were enrolled in the study between May and August of 2018. Dogs

belonging to faculty, staff, and students of the College, as well as patients of the Veterinary

77 Medicine Center, were eligible for enrollment. Prior to enrollment, informed consent was

78 obtained for each dog from the owner, along with information regarding known medical

79 conditions and current medications.

80 Dogs that were intolerant of the procedure, aggressive, or received sedation or anesthesia

81 within the previous 12 hours were excluded from the study. All other dogs, regardless of disease

82 status or medications received, were included to best approximate a clinical population and allow

83 comparison with prior studies (Bosiack et al. 2010; Hsiang et al. 2008; Mooney et al. 2017;

84 Wernick et al. 2012). 

generator (https://www.random.org, accessed 5/14/18) to determine whether subjects would

87

undergo blood pressure measurement with or without headphones first.

Data collection - All data collection was performed in a quiet examination room by 1 investigator (IRG). If the owner was unable to be present for the duration of the protocol, the process was either carried out by the investigator alone or with the help of a veterinary assistant who was present for the duration of the visit.

Weight, body condition score (BCS) and muscle condition score (MCS) (Baldwin et al. 2010), anxiety score (Scansen et al. 2014), and heart rate were recorded immediately upon entry into the room. The original American College of Veterinary Internal Medicine Consensus Statement guidelines (Brown et al. 2007) were followed for collection of blood pressure measurement, because the current guidelines (Acierno et al. 2018) had not been published at the time of study completion. Measurements were taken using the left radial artery with the dog in right lateral recumbency. If this position could not be used due to absence of a limb, injury, pain, or intravenous catheter location, the right forelimb was used with the animal in left lateral recumbency.

A soft measuring tape was used to measure the circumference of the mid-antebrachium, and a cuff was selected such that the width of the cuff was $30-40 \%$ of the limb circumference at the site of cuff placement. The palmar surface of the foot between the carpal and metacarpal pads was shaved for placement of the Doppler crystal. The dog then was allowed to acclimate to the environment and personnel for 10 minutes. Heart rate and anxiety score were recorded at the beginning and end of the acclimation period, as were acclimation start and stop times.

After the conclusion of the acclimation period, the investigator placed a blood pressure 
108 cuff on the mid-antebrachium with the dog in lateral recumbency and the forelimb held at the

109 level of the heart. Headphones were worn by the investigator when indicated by the

110 randomization system. The concave side of a flat infant Doppler probe was covered in ultrasonic

111 coupling gel and secured over the artery with bandage tape. The cuff was inflated to

112 approximately $20 \mathrm{mmHg}$ above the point at which blood flow could no longer be heard. Air was

113 slowly released until blood flow was audible, the value of which was recorded. Five more

114 readings were obtained in this manner. Heart rate and anxiety score were collected at the start

115 and stop of each measurement series, as were measurement start and stop times. Following the

116 first set of data collection, all equipment was removed and a second 10-minute acclimation

117 period started, with the procedure repeated with or without headphones as per the crossover 118 design.

119 The same Doppler unit and probe (Model 811-B Doppler ultrasonic Flow Detector with 120 flat infant probe; Parks Medical Electronics, Inc., Aloha, OR, USA), sphygmomanometer 121 (Riester Ri-san ${ }^{\circledR}$ aneroid sphygmomanometer; Riestar Direct, Ventura, CA, USA), blood 122 pressure cuffs (SunTechMed Soft Disposable Cuffs, Suntech Medical, Morrisville, NC, USA), 123 and headphones (Panasonic RP-HT161 Stereo Headphones, Suntech Medical, Morrisville, NC, 124 USA) were used for all subjects.

Data entry and statistical analyses - After each visit, data collection forms were submitted to a person unrelated to the study. This individual entered the data into a file that was unavailable to investigators until the conclusion of data collection. were analyzed for normality using the Shapiro-Wilk test and Q-Q plots. Each continuous 
131 evaluated using Levene's test for equality of variances. Parameters with normally distributed

132 data were reported as mean \pm standard deviation, with non-normal data reported as median

133 (range).

134 The number of dogs categorized as hypertensive based on blood pressure measurements

$135>150 \mathrm{mmHg}$ (Brown et al., 2007) was determined for each measurement type and period. The

136 paired sample student's t-test was used to compare the time required to complete data collection

137 for measurements collected with $v s$ without headphones.

138 Spearman rank correlation coefficients were used to assess correlations among BP 1, BP

$1392-6$, and possible covariates (age, weight, BCS, MCS, cuff size as a percent of limb

140 circumference; heart rate and anxiety score at the start of the measurement series; and time

141 required for collection of measurements) for each measurement type (headphone $v s$ non-

142 headphone). Spearman rank correlation coefficients and variance inflation factors from

143 regression analysis were used to assess for collinearity between possible covariates prior to

144 performing ANCOVA models.

145 A mixed effects crossover design with corresponding ANCOVAs were performed to

146 determine if BP 1 and BP 2-6 differed between headphone and non-headphone measurements

147 and to assess whether the washout period between measurement techniques was adequate (Littell

148 et al. 2006). Period and measurement type (headphone $v s$ non-headphone) were included as fixed

149 effects. Continuous measures with $\rho \leq 0.2$ on correlation testing were included as covariates in

150 the initial analysis. Covariates initially included in the ANCOVA models were age, BCS, MCS,

151 weight, as well as anxiety score and heart rate taken at the start of measurements. Dog nested

152 within sequence was included as a random effect. A compound symmetry variance/covariance

153 structure was incorporated into each model to account for constant covariates. Each covariate 
154 was tested for homogeneity of slopes between measurement types. Equal slopes ANCOVA

155 models were determined for each covariate (Littell et al. 2006). Backwards variable selection

156 was performed on the full models to determine which covariates explained significant variability

157 in results. Covariates included in the final models were period, measurement type (headphone vs

158 non-headphone), age, weight, and anxiety score. The Shapiro-Wilk test of normality of the

159 residuals and Levene's test for equality of variances was evaluated to ensure the assumptions of

160 the statistical method had been met. Commercial statistical software packages (SAS 9.4 release

161 TS1M5; SAS Institute Inc., Cary, NC, USA; IBM SPSS Statistics for Windows, version 25; IBM

162 Corp., Armonk, NY, USA) were used for all analyses. $P<0.05$ was considered significant.

\section{Results}

165 One hundred dogs initially were enrolled in the study (Supplementary file 1), 17 of which

were excluded (Figure 1). Of the remaining 83 dogs (Table 1), 48 dogs had measurements taken

167

168

169

170

171

172

173

174

175

176

using headphones first and 35 dogs had measurements taken without headphones first.

Demographic information and results of each blood pressure measurement series are listed in Table 1, while information for each dog is presented in Supplemental file 1. The most common breeds included were mixed breed dogs (39), Golden retriever (6), Labrador retriever (6), and Border Collie (3) with $\leq 2$ dogs of 27 other breeds. Fifty-eight dogs were categorized as healthy. The most common diseases identified in the remaining 25 dogs were categorized as metabolic (13), musculoskeletal (6), dermatologic (5), endocrine (4), and neurologic (3), with 13 dogs having $>1$ condition diagnosed. Seven dogs had been diagnosed with $\geq 1$ disease associated with hypertension: chronic kidney disease (4), hyperadrenocorticism (2), and diabetes mellitus with uncharacterized azotemia (1). Five dogs were receiving a medication that could potentially affect 
177 blood pressure results. Three dogs were receiving prednisone, 1 dog with chronic kidney disease

178 was receiving amlodipine, and a different dog with chronic kidney disease was receiving

179 phenylpropanolamine.

180 There was no difference in the number of dogs that would have been diagnosed as

181 hypertensive between measurement types (headphones, 19 dogs; without headphones, 18 dogs)

182 or periods (period 1, 21 dogs; period 2, 16 dogs). Seven dogs were categorized as hypertensive

183 during both periods. Time required to collect blood pressure measurements also did not differ

184 between measurement types (headphone, $4.9 \pm 2.3$ minutes; without headphones, $4.7 \pm 2.1$

185 minutes).

Correlation between BP 1 and BP 2-6 was almost perfect for measurements taken with ( $\rho$ 187 $=0.95, P<0.01)$ and without $(\rho=0.94, P<0.01)$ headphones. For headphones measurements, the median BP 1 for all dogs across both periods was $130 \mathrm{mmHg}$ (range, 80-210 $\mathrm{mmHg}$ ) while the median BP 2-6 was $133 \mathrm{mmHg}$ (range, 84-202 mmHg). For non-headphones measurements, 190 the median BP 1 for all dogs across both periods was $136 \mathrm{mmHg}$ (range, 90-270 $\mathrm{mmHg}$ ) while the median BP 2-6 was $131 \mathrm{mmHg}$ (range, 86-255 $\mathrm{mmHg}$ ).

192 With regard to potential covariates, BP 1 was significantly correlated with BCS for measurements taken with headphones $(\rho=0.24, P=0.03)$ but not for measurements taken without headphones. Conversely, BP 2-6 measurements were significantly correlated with age ( $\rho$ $=0.26, P=0.02)$ and $\operatorname{BCS}(\rho=0.26, P=0.02)$, respectively, for headphones measurements. Similar correlations were not found for BP 2-6 measurements taken without headphones. See Table 2 for significant associations identified among covariables. explain significant variability in the results were BCS, MCS, and heart rate. Thus, the final 
200 model included period, measurement type, age, weight, and anxiety score. There was no

201 significant effect of period. Significant differences in BP 1 results were found between

202 measurement type $(\mathrm{F}[1,80]=4.3, P=0.04)$. Post hoc analysis revealed that differences were due

203 to higher results when taken without headphones (5.4 mmHg increase in the marginal means).

204 Significant covariates affecting BP 1 were anxiety score $(\beta=9.5, \mathrm{t}(158)=3.4, P<0.01)$, age $(\beta$

$205=2.4, \mathrm{t}(83)=3.1, P<0.01)$, and weight $(\beta=0.4, \mathrm{t}(83)=3.4, P<0.01)$. Thus, for every 1 unit

206 increase in anxiety score, a $9.5 \mathrm{mmHg}$ increase was observed in BP 1 holding all other measures

207 constant. Similarly, for every year increase in age, BP 1 increased by $2.4 \mathrm{mmHg}$. For every $\mathrm{kg}$

208 increase in weight, BP 1 increased by $0.4 \mathrm{mmHg}$.

209 For BP 2-6, potential covariates removed from the final mixed effects model were BCS,

210 MCS, and heart rate. There was no significant effect of period on the model. Furthermore, there

211 was no significant association between BP 2-6 and headphone use. Significant covariates

212 affecting BP 2-6 were anxiety score $(\beta=9.7, \mathrm{t}(158)=3.6, P<0.01)$, age $(\beta=2.4, \mathrm{t}(83)=3.2, P$

$213<0.01)$, and weight $(\beta=0.4, \mathrm{t}(83)=3.1, P<0.01)$. Thus, for every 1 unit increase in anxiety

214 score, a $9.7 \mathrm{mmHg}$ increase was observed in BP 2-6, holding all other measures constant. For

215 every year increase in age, BP 2-6 increased by $2.4 \mathrm{mmHg}$. Finally, for every kg increase in

216 weight, BP 2-6 increased by $0.4 \mathrm{mmHg}$.

217

218 Discussion

219 In this study, no significant difference was identified between BP 2-6 results taken with

or without headphones in dogs. However, BP 1 results taken without the use of headphones were

221 significantly higher than results taken with headphones. Although some dogs with high anxiety 
223 associated with an increase of $>9 \mathrm{mmHg}$ in blood pressure overall. Finally, in spite of even

224 randomization between the two groups, 10 times as many dogs in the group randomized to first

225 have measurements taken without the use of headphones were excluded from the study due to

226 excessive anxiety. Thus, it is possible that the lack of association between headphone usage and

227 BP 2-6 results reflects self-elimination from blood pressure measurement by dogs most

228 vulnerable to white coat effect.

229

Another factor that might have influenced results is differing speaker quality between the

Doppler unit and headphones. It is possible that improved sound clarity, decreased static, and

231

232

233

234

235

236

237

238

239

240

241

242

243

244

245 reduction of ambient noise associated with using headphones allowed for earlier detection of the return of blood flow during cuff deflation. This could have offset any mitigating effects of headphone use on changes in pressure due to sounds emitted by the Doppler unit, particularly during patient movement.

The impact of age on blood pressure in dogs remains unclear (Acierno et al. 2018). In this study, blood pressure increased $2.4 \mathrm{mmHg}$ for every year of increasing age. These results are consistent with results of some, but not all, prior reports. Differences in associations among studies could reflect differences in study methodology or the population evaluated. For example, two studies identifying a significant positive association between age and blood pressure included age as a continuous variable in the analysis (Bodey \& Michell 1996; Bright \& Dentino 2002). In contrast, one study that found no association between age and blood pressure did not evaluate age as a continuous variable (Meurs et al. 2000). Dogs instead were categorized as adult $v s$ geriatric based on their age relative to their weight. Further, not all studies included assessment of anxiety or temperament in their analyses. In this study, age was inversely correlated with anxiety score, potentially reflecting an age-related decrease in excitability. 
246 Because they were inversely associated, failure to include both in the statistical model could

247 mask associations between blood pressure and either variable. Finally, screening of apparently

248 healthy dogs was not performed in this or prior reports for diseases associated with hypertension,

249 such as chronic hyperadrenocorticism, diabetes mellitus, or kidney disease. In one study that

250 found no association between age and blood pressure, approximately half of enrolled dogs had at

251 least one known disease (Mooney et al. 2017), but only $20 \%$ of them had a disease known to be

252 associated with hypertension. Positive associations in some studies, thus, could reflect age-

253 related vascular stiffening and loss of compliance (Acierno \& Labato 2004; Meurs et al. 2000) or

254 merely an increased prevalence of diseases associated with hypertension.

255 Consistent with prior reports (Acierno et al. 2018; Mooney et al. 2017), we found no

256 association between BCS and blood pressure in the mixed model analysis. The positive

257 correlation between BCS and blood pressure results on Spearman rank analysis, thus, can be

258 presumed to reflect confounding due to effects of subject age, weight, and anxiety score.

259 Blood pressure has previously been found to differ significantly among breeds, with

260 lower blood pressures found in larger breed dogs compared to smaller breed dogs (Höglund et al.

261 2012) and published references intervals (Bright \& Dentino 2002). The exception to this

262 association is sighthounds, which typically have higher blood pressures (Acierno et al. 2018). It

263 has been postulated that breed associations could reflect breed-associated temperament (Bodey

264 \& Michell 1996; Chetboul et al. 2010; Höglund et al. 2012; Schellenberg et al. 2007), but a calm

265 external demeanor was associated with lower blood pressures results among dogs of a single

266 breed (Bright \& Dentino 2002). It was not possible to directly assess the impact of breed on

267 results of this study, but weight and anxiety score were independently associated with blood 
268 pressure in this study. Taken as a whole, these results suggest that differences in blood pressure

269 among breeds are not entirely dependent on outward demeanor or temperament.

270 One concerning finding in this study was a lack of consistency in detection of

271 hypertension. Only 7 of 30 dogs categorized as hypertensive were so categorized in both

272 measurement periods, in spite of careful attention to optimal technique. The remainder had

273 results consistent with hypertension in only 1 of the 2 periods. This underscores the importance

274 of confirming abnormal results before pursuing treatment, particularly in patients without

275 evidence of end-organ damage or diseases associated with hypertension (Acierno et al. 2018).

276 Current veterinary guidelines recommend discarding the first of 5-7 indirect blood pressure

277 readings (Acierno et al. 2018). However, BP 1 and BP 2-6 results did not differ in dogs when

278 taken using either the coccygeal or radial artery and Doppler flow with headphones in one study

279 (Mooney et al. 2017). Two normotensive subjects in that report had past histories of refractory

280 hypertension due to inadequate acclimation to hospital personnel and equipment prior to blood

281 pressure measurement. Blood pressure measurement results for both remained normal after

282 clinical protocol was adjusted to ensure acclimation to personnel, resulting in discontinuation of

283 antihypertensive therapy. Based on those observations, the authors recommended that

284 acclimation of the subject to personnel and equipment be prioritized over collection of multiple

285 readings in cases where adequate time is not available for both. Given significantly higher BP 1

286 vs 2-6 results for measurements taken without headphones in this study, use of a solitary blood

287 pressure reading only could be considered for Doppler flow blood pressure measurements taken

288 using headphones.

289 The current recommendation is to use cuffs that are $30-40 \%$ of the antebrachium

290 circumference for radial indirect blood pressure measurement (Acierno et al. 2018). Use of 
291 inappropriately-sized cuffs was associated with over- and underestimating indirect blood

292 pressure when too small or too large a cuff was used, respectively, in one study of oscillometric

293 pressure measurements in dogs (Bodey et al. 1994). However, a similar association was not

294 identified in a more recent study of indirect Doppler flow collected measurements (Mooney et al.

295 2017). In this study, cuff size as a percentage of limb circumference was not correlated with

296 blood pressure results.

297

This study had a few additional limitations. First, the narrow range of the anxiety scoring

298

system used (Scansen et al. 2014) limited detection of smaller changes in demeanor.

299 Furthermore, this scale hinges on factors external to the animal, including how much restraint

300

was required and when the owner intervened - either of which could be affected by non-anxiety

301

related factors. To better differentiate patient anxiety from non-patient factors, it is recommended

302

that future studies evaluate anxiety using a scoring system that relies directly on changes in the

patient demeanor such as the Fear and Anxiety Scoring system (Fear Free LLC 2017).

304

Additionally, the study contained few dogs with diseases or medications that could affect blood

pressure results. The magnitude of the white-coat effect was significantly higher in cats with

306

experimentally-induced kidney disease compared to healthy cats in 1 study (Belew et al. 1999).

Thus, caution should be exercised when extrapolating results of this study to dogs with chronic

kidney disease, other diseases associated with hypertension, or receiving medications known to alter blood pressure. Further, the impact of potential habituation due to recurrent admission on results was not assessed. Both increased and decreased blood pressure were noted in healthy research cats undergoing repeated simulated office visits (Belew et al. 1999). Similarly, both

312 situational hypertension and masked hypertension have been identified in people (Acierno et al.

313 2018). Conversely, although there was no significant association between blood pressure results 
314 in greyhound blood donors and number of prior admissions for blood collection (Marino et al.

315 2011), oscillometric blood pressure results were significantly higher when results were taken in

316 the hospital $v s$ at home, regardless of whether home measurements were collected by the

317 investigator or the owner. A crossover design was not used for that study and details were

318 limited regarding the blood pressure measurement protocol, including factors such as length of

319 acclimation to the investigator in the hospital environment (the first measurement series taken).

320 Finally, it is unclear whether the correlation analyses between blood pressure results and number

321 of hospital visits were performed using measurements collected in hospital $v s$ at home. Thus, it is

322 unclear whether the significant difference in results between locations in the study by Marino et

323 al was due to hospital-induced white-coat effect, inadequate initial acclimation to the

324 investigator/protocol, or habituation to the measurement technique over time. Either of the latter

325 possibilities could have obscured an association between habituation to the hospital and blood

326 pressure results. Further evaluation using a crossover design might aid in clarifying the separate

327 effects of habituation to measurement personnel vs environment on white-coat effects in dogs.

\section{Conclusions}

Initial indirect Doppler systolic blood pressure measurements taken without headphones were significantly higher in dogs than measurements taken using headphones. This effect did not persist for BP 2-6 measurements, which could reflect acclimation to the experience. Conversely, exclusion of 10 times as many dogs from the trial due to anxiety when measurements were first taken without headphones could have masked a significant association between BP 2-6 results and blood pressure measurement type. This possibility is supported by the strong association identified between anxiety score and blood pressure results in this study. Pending further 
337

338

339

340

341

342

343

344

345

346

347

348

349

350

351

352

353

354

355

356

357

358

359

360

361

362

363

364

365

366

367

368

369

370

371

372

373

374

375

evaluation using a larger sample size, it is prudent to use headphones for indirect systolic blood

pressure measurement determination to avoid erroneous diagnosis of hypertension in dogs.

\section{References}

Acierno MJ, Brown S, Coleman AE, Jepson RE, Papich M, Stepien RL, and Syme HM. 2018. ACVIM consensus statement: Guidelines for the identification, evaluation, and management of systemic hypertension in dogs and cats. Journal of Veterinary Internal Medicine 32:1803-1822. 10.1111/jvim.15331

Acierno MJ, and Labato MA. 2004. Hypertension in dogs and cats. Compend Cont Ed Pract Vet 26:336-346.

Baldwin K, Bartges J, Buffington T, Freeman LM, Grabow M, Legred J, and Ostwald DJ. 2010. AAHA nutritional assessment guidelines for dogs and cats. Journal of the American Animal Hospital Association 46:285-296.

Belew AM, Barlett T, and Brown SA. 1999. Evaluation of the white-coat effect in cats. Journal of Veterinary Internal Medicine 13:134-142.

Bodey AR, and Michell AR. 1996. Epidemiological study of blood pressure in domestic dogs. Journal of Small Animal Practice 37:116-125.

Bodey AR, Young LE, Bartram DH, Diamond MJ, and Michell AR. 1994. A comparison of direct and indirect (oscillometric) measurements of arterial blood pressure in anaesthetised dogs, using tail and limb cuffs. Research in Veterinary Science 57:265-269.

Bosiack AP, Mann FA, Dodam JR, Wagner-Mann CC, and Branson KR. 2010. Comparison of ultrasonic Doppler flow monitor, oscillometric, and direct arterial blood pressure measurements in ill dogs. J Vet Emerg Crit Care 20:207-215.

Bragg RF, Bennett JS, Cummings A, and Quimby JM. 2015. Evaluation of the effects of hospital visit stress on physiologic variables in dogs. Journal of the American Veterinary Medical Association 246:212-215.

Bright JM, and Dentino M. 2002. Indirect Arterial Blood Pressure Measurement in Nonsedated Irish Wolfhounds: Reference Values for the Breed. Journal of the American Animal Hospital Association 38:521-526. 10.5326/0380521

Brown S, Atkins C, Bagley R, Carr A, Cowgill L, Davidson M, Egner B, Elliott J, Henik R, Labato M, Littman M, Polzin D, Ross L, Snyder P, and Stepien R. 2007. Guidelines for the identification, evaluation, and management of systemic hypertension in dogs and cats. Journal of Veterinary Internal Medicine 21:425-558.

Caney SMA. 2007. Non-invasive blood pressure measurement in cats. In Practice 29:398-403. 10.1136/inpract.29.7.398

Chetboul V, Tissier R, Gouni V, de Almeida V, Lefebvre HP, Concordet D, Jamet N, Sampedrano CC, Serres F, and Pouchelon J-L. 2010. Comparison of Doppler ultrasonography and highdefinition oscillometry for blood pressure measurements in healthy awake dogs. American Journal of Veterinary Research 71:766-772. 10.2460/ajvr.71.7.766 
376

377

378

379

380

381

382

383

384

385

386

387

388

389

390

391

392

393

394

395

396

397

398

399

400

401

402

403

404

405

406

407

408

409

410

411

412

413

414

Fear Free LLC. 2017. Fear, Anxiety, and Stress Spectrum. Available at https://fearfreepets.com/fas-spectrum/ (accessed 2018-07-22 2018).

Höglund K, Hanås S, Carnabuci C, Ljungvall I, Tidholm A, and Häggström J. 2012. Blood pressure, heart rate, and urinary catecholamines in healthy dogs subjected to different clinical settings. Journal of Veterinary Internal Medicine 26:1300-1308.

Hsiang TY, Lien YH, and Huang HP. 2008. Indirect measurement of systemic blood pressure in conscious dogs in a clinical setting. Journal of Veterinary Medical Science 70:449-453.

Littell RC, Milliken GA, Stroup WW, Wolfinger RD, and Schanbenberger O. 2006. SAS for Mixed Models, Second Edition. Cary, NC: SAS Institute Inc.

Marino CL, Cober RE, lazbik MC, and Couto CG. 2011. White-Coat Effect on Systemic Blood Pressure in Retired Racing Greyhounds. Journal of Veterinary Internal Medicine 25:861865. 10.1111/j.1939-1676.2011.00735.x

Meurs KM, Miller MW, Slater MR, and Glaze K. 2000. Arterial blood pressure measurement in a population of healthy geriatric dogs. Journal of the American Animal Hospital Association 36:497-500. 10.5326/15473317-36-6-497

Mooney AP, Mawby DI, Price JM, and Whittemore JC. 2017. Effects of various factors on Doppler flow ultrasonic radial and coccygeal artery systolic blood pressure measurements in privately-owned, conscious dogs. PeerJ 5:e3101. 10.7717/peerj.3101

Rondeau DA, Mackalonis ME, and Hess RS. 2013. Effect of body position on indirect measurement of systolic arterial blood pressure in dogs. Journal of the American Veterinary Medical Association 242:1523-1527.

Scansen BA, Vitt J, Chew DJ, Schober HE, and Bonagura JD. 2014. Comparison of forelimb and hindlimb systolic blood pressures and proteinuria in healthy Shetland Sheepdogs. Journal of Veterinary Internal Medicine 28:277-283.

Schellenberg S, Glaus TM, and Reusch CE. 2007. Effect of long-term adaptation on indirect measurements of systolic blood pressure in conscious untrained beagles. Veterinary Record 161:418. 10.1136/vr.161.12.418

Wernick MB, Höpfner RM, Francey T, and Howard J. 2012. Comparison of arterial blood pressure measurements and hypertension scores obtained by use of three indirect measurement devices in hospitalized dogs. Journal of the American Veterinary Medical Association 240:962-968.

Whittemore JC, Nystrom MR, and Mawby DI. 2017. Effects of various factors on Doppler ultrasonographic measurements of radial and coccygeal arterial blood pressure in privately owned, conscious cats. Journal of the American Veterinary Medical Association 250:763-769. 10.2460/javma.250.7.763

Williams TL, Elliot J, and Syme HM. 2010. Measurement of systolic blood pressure (SBP) in cats by the indirect Doppler technique is not altered by the use of headphones. ECVIM Congress. 


\section{Table $\mathbf{1}$ (on next page)}

Demographics and indirect Doppler systolic arterial blood pressure readings collected with and without headphones using a crossover design for 83 privately-owned dogs.

Values are reported as median \pm standard deviation. F, Female; FS, female spayed; M, male; MC, male castrated; BCS, body condition score on a scale of 1-9; MCS, muscle condition score on a scale of 1-3; BP, blood pressure. 


\begin{tabular}{|c|c|c|}
\hline & $\begin{array}{l}\text { Headphones, } \\
\text { no headphones }\end{array}$ & $\begin{array}{c}\text { No headphones, } \\
\text { headphones }\end{array}$ \\
\hline \multicolumn{3}{|l|}{ Sex } \\
\hline$-\quad F, F S$ & 4,20 & 0,15 \\
\hline - $M, M C$ & 4,20 & 3,17 \\
\hline Age (years) & $5.6 \pm 3.4$ & $5.16 \pm 3.0$ \\
\hline BCS & $5.5 \pm 1.1$ & $5.4 \pm 1.2$ \\
\hline MCS & $2.9 \pm 0.4$ & $2.9 \pm 0.3$ \\
\hline Weight $(\mathrm{kg})$ & $22.5 \pm 14.9$ & $27.4 \pm 22.7$ \\
\hline \multicolumn{3}{|l|}{ First measurement period } \\
\hline - Anxiety score & $1.6 \pm 0.7$ & $1.7 \pm 0.9$ \\
\hline - $\mathrm{BP} 1(\mathrm{mmHg})$ & $135 \pm 25$ & $144 \pm 35$ \\
\hline - Mean of BP 2-6 (mmHg) & $134 \pm 21$ & $141 \pm 33$ \\
\hline \multicolumn{3}{|l|}{ Second measurement period } \\
\hline - Anxiety score & $1.4 \pm 0.7$ & $1.6 \pm 0.9$ \\
\hline - $\mathrm{BP} 1(\mathrm{mmHg})$ & $136 \pm 23$ & $135 \pm 26$ \\
\hline - Mean of BP 2-6 $(\mathrm{mmHg})$ & $136 \pm 24$ & $135 \pm 27$ \\
\hline
\end{tabular}




\section{Table 2 (on next page)}

Spearman rank correlation coefficients ( $\rho$ ) among covariables for 83 privately-owned dogs in which indirect Doppler systolic arterial blood pressure readings were collected with and without headphones.

MCS, muscle condition score on a scale of 1-3; ns, not significant. 


\begin{tabular}{|c|c|c|}
\hline & Headphones & Without headphones \\
\hline \multicolumn{3}{|l|}{ Anxiety score } \\
\hline$-\quad$ Age & $\rho=-0.23, P=0.04$ & $\rho=-0.26, P=0.02$ \\
\hline - Weight & $\rho=-0.23, P=0.03$ & $\rho=-0.25, P=0.02$ \\
\hline - $\quad$ Heart rate & $\rho=0.34, P<0.01$ & $\rho=0.38, P<0.01$ \\
\hline - $\quad$ MCS & $\rho=0.30, P=0.01$ & $\rho=0.22, P=0.04$ \\
\hline - Total measurement time & $\rho=0.25, P=0.02$ & $\rho=0.23, P=0.04$ \\
\hline \multicolumn{3}{|l|}{ Age } \\
\hline$-\quad M C S$ & $\rho=-0.39, P<0.01$ & $\rho=-0.39, P<0.01$ \\
\hline - $\quad$ Anxiety score & $\rho=-0.23, P=0.04$ & $\rho=-0.25, P=0.02$ \\
\hline \multicolumn{3}{|l|}{ Heart rate } \\
\hline - Weight & $\rho=-0.29, P=0.01$ & $\rho=-0.39, P<0.01$ \\
\hline - MCS & $\rho=0.30, P=0.01$ & $\rho=0.19, P=0.08$ \\
\hline - $\quad$ Anxiety score & $\rho=0.36, P<0.01$ & $\rho=0.38, P<0.01$ \\
\hline \multicolumn{3}{|l|}{ Total measurement time } \\
\hline - MCS & ns & $\rho=0.23, P=0.04$ \\
\hline - $\quad$ Anxiety score & $\rho=0.25, P=0.02$ & $\rho=0.23, P=0.04$ \\
\hline
\end{tabular}


Figure 1

Flowchart showing the distribution of 100 privately-owned dogs randomized to undergo collection of systolic blood pressure measurements with and without headphones using a crossover design.

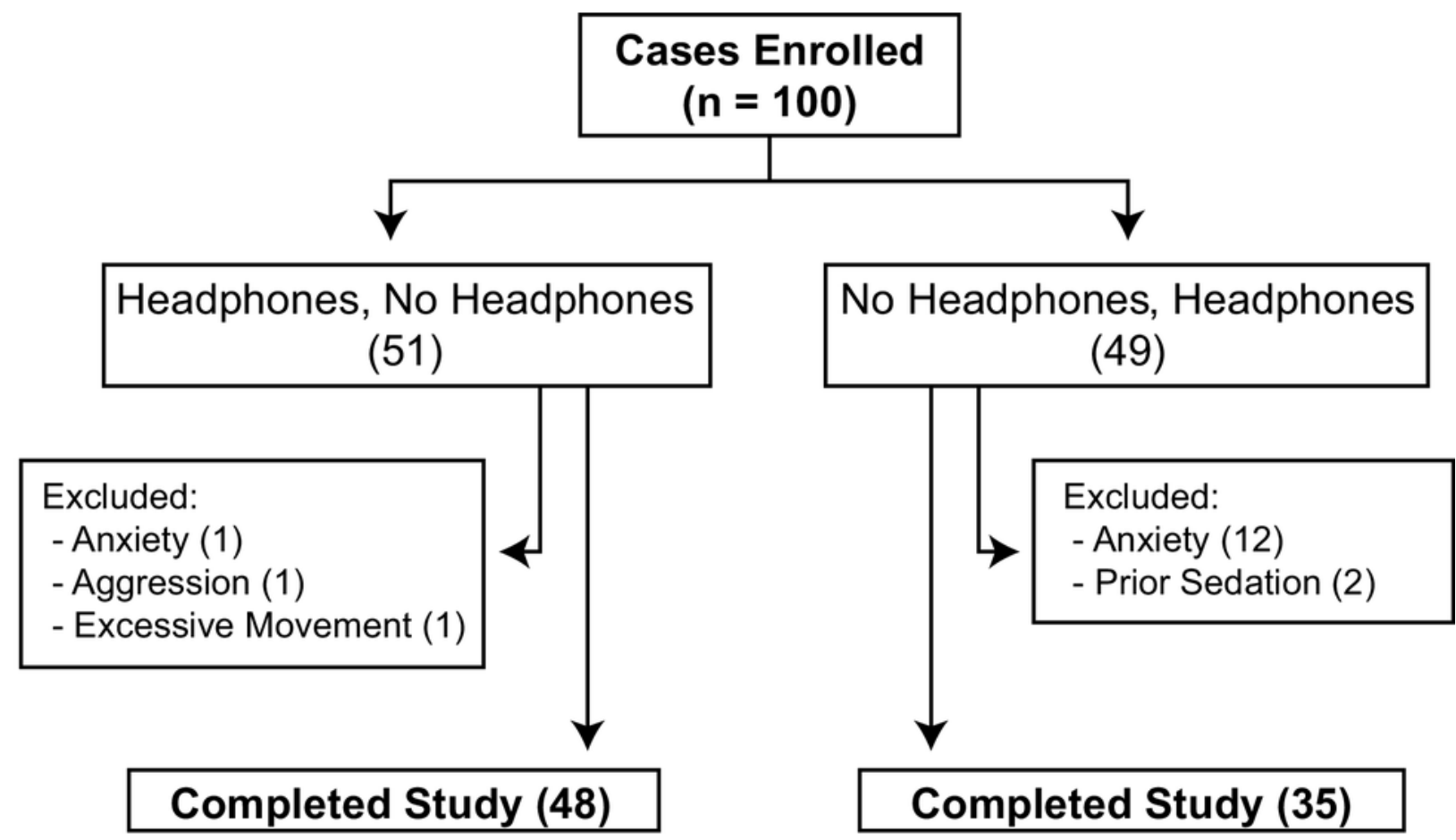

